Pacific Journal of Mathematics

ON CENTRAL TYPE FACTOR GROUPS 


\title{
ON CENTRAL TYPE FACTOR GROUPS
}

\section{SHAHRIAR SHAHRIARI}

\begin{abstract}
$\Gamma$ is a group of central type if it possesses an irreducible complex character of degree $|\Gamma: Z(\Gamma)|^{1 / 2}$. This is the largest possible degree for an ordinary irreducible character of a finite group. A group $G$ which is isomorphic to $\Gamma / \mathrm{Z}(\Gamma)$, where $\Gamma$ is some group of central type, is called a central type factor group (ctfg). A variety of restrictions on ctfgs are found. These include a local characterization of ctfgs, and restrictions on normal and subnormal structures of ctfgs.
\end{abstract}

1. Introduction. It is easy to see (see Corollary 2.30 of [10]) that the degree of an irreducible character of a finite group $\Gamma$ cannot be larger than $|\Gamma: \mathbf{Z}(\Gamma)|^{1 / 2}$. A group $\Gamma$ that has an irreducible character of this maximal degree is called a group of central type. A group $G$ which is isomorphic to $\Gamma / \mathbf{Z}(\Gamma)$, where $\Gamma$ is some group of central type, is called a central type factor group (ctfg for short).

A configuration that occurs often in character theory of solvable groups, and therefore has been the object of much research, is that of fully ramified sections (see [4], [6], [8], [9], [14]). A normal subgroup $N$ of $\Gamma$ is said to be fully ramified in $\Gamma$, if there exists an irreducible character $\theta$ of $N$, such that $\theta^{\Gamma}=e \chi$, for $\chi$ some irreducible character of $G$, and $\chi_{N}=e \theta$. Now, if $N$ is fully ramified in $\Gamma$, then $\Gamma / N$ is a ctfg (see Lemma 2.6), and thus the study of fully ramified sections reduces to that of ctfgs. Another reason why characterizations of ctfgs are sought after is that a group $G$ is a ctfg if and only if it possesses a 2-cocycle $\alpha$ such that the twisted group algebra $\mathbb{C}^{\alpha}[G]$ is simple (see Theorem 2.7).

The study of these groups goes back to Iwahori and Matsumoto in 1964. They conjectured that ctfgs must be solvable (see [11]). Various properties of ctfgs were discovered in [3], [4], and [12]. In 1982, Howlett and Isaacs [6] proved the solvability of ctfgs.

The problem of understanding solvable ctfgs remains open. In this paper, we will get more restrictions on the structure of a ctfg.

DeMeyer and Janusz [3] proved that if $G$ is a ctfg then so are its Sylow subgroups. However, to get that $G$ is a ctfg it is not enough to know that its Sylow subgroups are ctfg. DeMeyer and Janusz do provide enough additional conditions to assure that $G$ is a ctfg. 
However, their conditions are not local, that is, to know whether a group $G$ is a ctfg they still need certain information about the whole group $G$. Using the techniques of group cohomology, the following local characterization of ctfgs is obtained.

THEOREM A. A group $G$ is a ctfg if and only if every Sylow $p$ subgroup $P$ of $G$ has a 2-cocycle $\alpha$ such that $\mathbb{C}^{\alpha}[P]$ is simple and $[\alpha]$ is stabilized by $\mathbf{N}_{G}(Q)$ for all $1 \neq Q \subseteq P$.

The same techniques are applied to give the following characterization of ctfgs of odd order with abelian Sylow subgroups:

THEOREM B. A group $G$ of odd order is a ctfg with abelian Sylows if and only if for every $P \in \operatorname{Syl}_{p}(G)$ there is a nondegenerate alternating bilinear map $\alpha: P \times P \rightarrow \mathbb{C}^{\times}$which is preserved by $\mathbf{N}_{G}(P)$.

S. Gagola [4] has proved that any solvable group can be a subgroup of a ctfg. This severely limits the restrictions one might hope to put on ctfgs, since most conditions carry over to subgroups. In proving the solvability of groups of central type, Howlett and Isaacs [6] found some restrictions on normal subgroups of ctfgs. This, of course, is in sharp contrast to Gagola's result. Given these two results a natural question is whether there is any restrictions on subnormal subgroups of ctfgs. This question was answered in the affirmative. In fact we prove that a certain explicit set of groups cannot occur as subnormal subgroups of ctfgs.

THEOREM C. Let $S$ be the nonabelian group of order $p q$, where $p$ and $q$ are primes with $p \mid q-1$. Then $S$ cannot be a subnormal subgroup of a ctfg.

The study of the normal structure of a ctfg is continued by proving several restrictions on possible normal subgroups of a ctfg. These include:

TheOREM D. Assume that $G$ is a ctfg, and $S$ is a noncyclic normal subgroup of $G$. Then the Schur Multiplier of $S, M(S)$, and the Schur Multiplier of the Fitting subgroup of $S, M(\mathbf{F}(S))$, are not trivial.

Theorem E. Let $S$ be the semi-direct product $N \rtimes H$. Assume $(|N|,|H|)=1$, and $\mathbf{C}_{N}(H)=1$. Then $S$ is a normal subgroup of $a$ 
ctfg if and only if

(a) $N$ has a 2-cocycle $\alpha$ with $\mathbb{C}^{\alpha}[N]$ simple (i.e. $N$ is a ctfg).

(b) $H$ is a normal subgroup of a ctfg $G_{0}$.

(c) $G_{0}$ acts on $N$ with $H$ acting as in $S$, and $\alpha$ admits $G_{0}$.

Theorem F. Assume $A$ is a direct summand of a ctfg. Then $A$ is ctfg by abelian.

The definition of a ctfg raises questions about two related configurations: Which groups are isomorphic to $\Gamma / \mathbf{Z}(\Gamma)$ for some finite group $\Gamma$ ? These groups are called capable and were studied in [13]. Restrictions on capable groups result on further restrictions on ctfgs. For example, let $Q$ be a generalized quaternion group of order $2^{n}$ $(n>2)$, or a semidihedral group of order $2^{n} \quad(n>3)$, or an extraspecial group of order $p^{3}$ with exponent $p^{2}(p>2)$. Assume $Q \triangleleft S$. Then it follows from the results of [13] that $S$ cannot be a ctfg, and also $S$ cannot be a system normalizer of a ctfg.

The second related configuration is that of fully ramified subgroups that are not necessarily normal. This occurs when the restriction of an irreducible character to a subgroup (not necessarily normal) is the multiple of a single irreducible character, and the ratio of the degrees of these two characters is the square root of the index of the subgroup. Restrictions on these fully ramified subgroups, and their consequences for ctfgs, were discussed in [14].

We have also included a brief exposition of bilinear pairings on pairs of groups. The results are part of the folklore, and are usually presented in a vector space context. However the field free approach presented here can be helpful when studying central extensions.

2. Definitions and preliminaries. In this section we will first establish some notation, and then present some definitions and known facts that will be needed in what follows. We need to use some elementary facts from group cohomology. However, we will limit our attention to two dimensional cohomology groups with trivial action, since these are all that will be needed. Most of the facts mentioned are true in much more generality. All groups considered are finite, except perhaps the cohomology coefficient groups.

For any group $G$ and an abelian group $A$, let $Z(G, A), B(G, A)$ and $H(G, A)$ denote $Z^{2}(G, A), B^{2}(G, A)$ and $H^{2}(G, A)$ respectively. The action of $G$ on $A$ will be trivial. In fact most of the time we will take $A$ to be $\mathbb{C}^{\times}$, the multiplicative group of nonzero complex numbers. If $\alpha$ is a cocycle of $G$ (that is, $\alpha \in Z(G, A)$ ), then 
we denote by $[\alpha]$ the image of $\alpha$ in $H(G, A)$ under the canonical homomorphism. That is $[\alpha] \in H(G, A)$ is the coset of $B(G, A)$ that contains $\alpha$.

If $K \subseteq G$, and $\alpha$ is a cocycle of $G$ (that is, $\alpha \in Z(G, A)$ ), then via the usual restriction map we get an element of $Z(K, A)$. This map can be viewed as a homomorphism on $H(G, A)$ when appropriate. We denote the image of $\alpha$ under this map by $\alpha_{k}$. Similarly, the corestriction or transfer is a homomorphism from $H(K, A)$ to $H(G, A)$. If $\alpha$ is in the domain of this map then its image will be denoted by $\alpha^{G}$. This is not the standard notation, and is chosen to emphasize the similarity between statements in group cohomology and those in character theory.

Let $K$ be a subgroup of $G$ and $\alpha$ be an element of $Z(K, A)$. Then an element $\alpha^{g}$ of $Z\left(K^{g}, A\right)$ is defined by $\alpha^{g}\left(u^{g}, v^{g}\right)=\alpha(u, v)$ for $u$ and $v$ elements of $K$ and any $g \in G$. For $[\alpha] \in H(K, A)$ define $[\alpha]^{g} \in H\left(K^{g}, A\right)$ by $[\alpha]^{g}=\left[\alpha^{g}\right]$. This is well defined by Problem 6 p. 225 of $[\mathbf{1 5}]$. We say that $[\alpha]$ is stable in $G$ if $[\alpha]$ and $[\alpha]^{g}$ are the same when restricted to $K \cap K^{g}$ for all $g \in G$.

A lot is known about the concepts defined above; however all we need is their existence and the few facts listed below. For an exposition and proof see [15] and [1].

(2.1) Proposition. Let $G$ be any group and $K$ and $L$ be subgroups of $G$.

(i) Suppose $\alpha \in H(G, A)$. Then $\alpha_{K}$ is stable in $G$.

(ii) Suppose $\beta \in H(K, A)$ is stable in $G$, and $K$ has finite index in $G$. Then $\left(\beta^{G}\right)_{K}=|G: K| \beta$.

(iii) Suppose $K^{g} \cap L$ is trivial for all $g \in G$, and $\beta \in H(K, A)$. Then $\left(\beta^{G}\right)_{L}=0$.

(iv) Suppose $G$ is a finite $\pi$-group where $\pi$ is a set of primes. Then $H(G, A)$ is a $\pi$-group.

Proof. (i) is 40.3 in [1]. Parts (ii) and (iii) follow from the double coset formula (Mackey's Theorem) 40.2 in [1], and (iv) is Corollary 2 of 7.26 in [15].

The second cohomology group $H^{2}\left(G, \mathbb{C}^{\times}\right)$is also known as the Schur Multiplier and is denoted by $M(G)$. This group plays a substantial role in the theory of group extensions. We only need to know 
the following:

(2.2) Proposition. (i) Let $G$ be a group with $n$ generators and $n$ relations, then $M(G)=1$. In particular the Schur multiplier of the cyclic group and the generalized quaternion group is trivial. Also the Schur multiplier of a group with cyclic sylows is trivial. (ii) Let $G$ be an abelian group, with $G=A_{1} \times A_{2} \times \cdots \times A_{n}$ where each $A_{i}$ is cyclic of order $a_{i}$. Let $a_{i j}=\operatorname{gcd}\left(a_{i}, a_{j}\right)$, and let $A_{i j}$ be a cyclic group of order $a_{i j}$. Then $M(G)$ is the direct product of $\left\{A_{i j} \mid 1 \leq i<j \leq n\right\}$.

Proof. (i) is Theorem 25.2 and 25.3 of [7], and (ii) is Problem 4, p. 264 of [15].

We also need to introduce the complex twisted group algebra, $\mathbb{C}^{\alpha}[G]$. Let $G$ be a finite group and let $\alpha \in Z\left(G, \mathbb{C}^{\times}\right)$. Then $\mathbb{C}^{\alpha}[G]$ is the $\mathbb{C}$-vector space with basis $(\bar{g} \mid g \in G)$. Define multiplication in $\mathbb{C}^{\alpha}[G]$ by $\bar{g} \cdot \bar{h}=\overline{g h} \alpha(g, h)$ and extend via the distributive law. It is easily verified that $\mathbb{C}^{\alpha}[G]$ is a finite dimensional algebra. In fact (Problem 11.7 of $[\mathbf{1 0}]) \mathbb{C}^{\alpha}[G]$ is semisimple. Now if $\alpha$ and $\beta$ are equivalent, that is, they belong to the same coset of $B\left(G, \mathbb{C}^{\times}\right)$in $Z\left(G, \mathbb{C}^{\times}\right)$, then $\mathbb{C}^{\alpha}[G]$ and $\mathbb{C}^{\beta}[G]$ are isomorphic (Problem 11.1 of $[\mathbf{1 0}]$ ). Thus we can think of $\mathbb{C}^{\alpha}[G]$ as having been defined for $[\alpha] \in H\left(G, \mathbb{C}^{\times}\right)$.

We are interested in restrictions on the group $G$ if $G$ has a 2-cocycle $\alpha$ such that $\mathbb{C}^{\alpha}[G]$ is simple. Since $\mathbb{C}^{\alpha}[G]$ is a semisimple algebra, it is simple if and only if the vector space dimension of its center is one. Now let $\alpha$ be a 2-cocycle of $G$; we say that $g \in G$ is $\alpha$-special if $\alpha(g, c)=\alpha(c, g)$ for all $c \in \mathbf{C}_{G}(g)$. It is easily seen (Problem 11.4 of [10]) that if $g$ is $\alpha$-special then so is every conjugate of $g$ in $G$. Also if $\alpha$ and $\beta$ are equivalent then $g \in G$ is $\alpha$-special if and only if it is $\beta$-special. Thus we can speak of $[\alpha]$-special classes of $G$. Because of the following lemma the $\alpha$-special classes are relevant to our discussion.

(2.3) Lemma. Let $\alpha \in Z\left(G, \mathbb{C}^{\times}\right)$. Then the dimension of the center of $\mathbb{C}^{\alpha}[G]$ is equal to the number of conjugacy classes of $\alpha$-special elements of $G$. In particular, $\mathbb{C}^{\alpha}[G]$ is simple if and only if the identity is the only $\alpha$-special element of $G$.

Proof. This is problem 11.8 of [10].

The pair of groups $(\Gamma, N)$ is called a central extension by $G$ if $N \subseteq \mathbf{Z}(\Gamma)$ and $\Gamma / N$ is isomorphic to $G$. The following notation will be fixed for the rest of the paper: If $(\Gamma, N)$ is a central extension by 
$G$ then for $H \subseteq G$ define $H^{+}$to be the inverse image of $H$ under the canonical homomorphism from $\Gamma$ to $G$, which means $N \subseteq H^{+} \subseteq \Gamma$, and $H^{+} / N$ is isomorphic to $H$.

In Lemma 2.3 we saw that the dimension of the center of a twisted group algebra $\mathbb{C}^{\alpha}[G]$ is the same as the number of $\alpha$-special classes. The next result will relate this number to the ordinary character theory of a central extension by $G$ (we use the notation $\operatorname{Irr}(\Gamma \mid \theta)$ to denote the set of irreducible constituents of $\theta^{\Gamma}$ where $\theta$ is an irreducible character of some subgroup of $\Gamma$ ).

(2.4) Proposition. Let $(\Gamma, N)$ be a central extension by $G$. Let $X$ be a set of coset representatives for $N$ in $\Gamma$ and write $X=\left\{x_{g} \mid\right.$ $g \in G\}$ where $x_{g} \in \Gamma$ is in the inverse image of $g$ under the canonical homomorphism. Define $\tilde{\alpha}: G \times G \rightarrow N$ by $x_{g} x_{h}=\tilde{\alpha}(g, h) x_{g h}$. Then $\tilde{\alpha} \in Z(G, N)$, and the equivalence class of $\tilde{\alpha}$ is independent of the choice of $X$. Furthermore, if $\lambda$ is a linear character of $N$ define $\alpha(g, h)=\lambda(\tilde{\alpha}(g, h))$. Then $\alpha \in Z\left(G, \mathbb{C}^{\times}\right)$and the number of $\alpha$ special classes of $G$ is equal to $|\operatorname{Irr}(\Gamma \mid \lambda)|$. In the case that $[\alpha]=1$, the character $\lambda$ extends to $\Gamma$.

Proof. The first part is Lemma 11.9 of [10]. The second part follows from Problem 11.6, 11.7, and 11.8 of $[10]$. The case that $[\alpha]=1$ follows from Theorem 11.7 of [10]. A straightforward calculation shows that the $[\beta]$ used in that theorem and constructed in the proof of Theorem 11.2 is the inverse of our $[\alpha]$ in $H\left(G, \mathbb{C}^{\times}\right)$.

A subgroup $N$ of $\Gamma$ is said to be fully ramified in $\Gamma$ if there exists $\theta \in \operatorname{Irr}(N)$ with $\theta^{\Gamma}=e \chi$, and $\chi_{N}=e \theta$, for some $\chi \in \operatorname{Irr}(\Gamma)$. In such a situation we also say that $\theta$ is fully ramified in $\Gamma, \chi$ is fully ramified over $N$ and $\chi$ is fully ramified over $\theta$.

In this paper we only consider normal fully ramified subgroups. For a discussion of the more general case see [14]. The following lemma is easy to prove, and gives various characterizations of fully ramified characters:

(2.5) Lemma. Let $N$ be a normal subgroup of $\Gamma, \theta \in \operatorname{Irr}(N)$, $\chi \in \operatorname{Irr}(\Gamma \mid \theta)$; then the following are equivalent:

(i) $\theta^{\Gamma}=e \chi$, and $\chi_{N}=e \theta$ (that is $\chi$ is fully ramified over $\theta$ ).

(ii) $\theta^{\Gamma}=e \chi$, and $e^{2}=|\Gamma: N|$.

(iii) $\chi_{N}=e \theta$, and $e^{2}=|\Gamma: N|$.

(iv) $\chi$ vanishes off $N$ and $\chi_{N}$ is a multiple of $\theta$.

(v) $\chi$ vanishes off $N$ and $\chi(1)^{2}=|\Gamma: N| \theta(1)^{2}$. 
A group is said to be of central type if it is fully ramified over its center. A group that is isomorphic to $\Gamma / Z(\Gamma)$, where $\Gamma$ is a group of central type is said to be a central type factor group, or ctfg for short.

The next lemma shows that to study restrictions on groups $\Gamma / N$ where $N$ is fully ramified in $\Gamma$, it is enough to look only at ctfgs.

(2.6) Lemma. Suppose that $N$ is normal and fully ramified in $\Gamma$. Then $\Gamma / N$ is a ctfg.

Proof. This is Lemma 4.3 of [6].

In the light of Proposition 2.3, we mention the close connection between simple twisted group algebras and ctfgs that will be needed later.

(2.7) Theorem. A group $G$ is a ctfg if and only if it possesses a 2-cocycle $\alpha$ such that the twisted group algebra $\mathbb{C}^{\alpha}[G]$ is simple.

Proof. This is Theorem 1 of [3]. One direction is immediate from Proposition 2.4, and Lemma 2.3. The other direction follows after looking at a Schur representation group for $G$.

We will also need:

(2.8) Theorem (Howlett and Isaacs). A ctfg must be solvable.

Proof. This is Theorem 7.3 of [6].

3. Bilinear pairings on groups. In this section we will bring together some facts about bilinear pairings on groups. Some of these facts will be used in the subsequent discussion, and most of them are known as a part of the folklore, albeit in a slightly different form.

Let $H$ and $K$ be any groups, and let $A$ be an abelian group. We say that a map $\alpha$ from $H \times K$ into $A$ is a bilinear pairing if

$$
\alpha\left(h h^{\prime}, k\right)=\alpha(h, k) \alpha\left(h^{\prime}, k\right) \text { for all } h, h^{\prime} \in H \text { and } k \in K
$$

and

$$
\alpha\left(h, k k^{\prime}\right)=\alpha(h, k) \alpha\left(h, k^{\prime}\right) \quad \text { for all } h \in H \text { and } k, k^{\prime} \in K .
$$

Given $\alpha$ a bilinear pairing from $H \times K$ into $A$ and $L$ a subgroup of $H$ define $L^{\perp}=\{x \in K \mid \alpha(l, x)=1$ for all $l \in L\}$. Note that $L^{\perp}$ is a subgroup of $K$. Similarly for any $J \subseteq K$ define $J^{\perp}=\{x \in H \mid \alpha(x, j)=1$ for all $j \in J\}$. We say that $\alpha$ is left nondegenerate if $K^{\perp}=1$, and is right nondegenerate if $H^{\perp}=1 . \alpha$ is called nondegenerate if it is both left and right nondegenerate. 
(3.1) Lemma. Suppose $\alpha$ is a bilinear pairing from $H \times K$ into an abelian group $A$. Then the commutator subgroup $H^{\prime}$ is contained in $K^{\perp}$, and $K^{\prime}$ is contained in $H^{\perp}$. In particular $H / K^{\perp}$ and $K / H^{\perp}$ are abelian, and if $\alpha$ is left nondegenerate then $H$ is abelian.

Proof. For $k \in K$ define a homomorphism $\phi_{k}$ from $H$ into $A$ by $\phi_{k}(h)=\alpha(h, k)$. This is a homomorphism into an abelian group and therefore $H / \operatorname{ker}\left(\phi_{k}\right)$ is abelian. It follows that $H^{\prime} \subseteq \operatorname{ker}\left(\phi_{k}\right)$ for all $k \in K$. However $K^{\perp}$ is the intersection of all the $\operatorname{ker}\left(\phi_{k}\right)$ for $k \in K$, and thus $H^{\prime} \subseteq K^{\perp}$. Similarly $K^{\prime} \subseteq H^{\perp}$.

Given $A$ and $B$ abelian groups, we let $\operatorname{Hom}(A, B)$ be the group of homomorphisms from $A$ into $B$ with pointwise multiplication.

(3.2) Proposition. Suppose $\alpha$ is a bilinear pairing from $H \times K$ into an abelian group $A$. Then $K / H^{\perp}$ is isomorphic to a subgroup of $\operatorname{Hom}\left(H / K^{\perp}, A\right)$, and $H / K^{\perp}$ is isomorphic to a subgroup of $\operatorname{Hom}\left(K / H^{\perp}, A\right)$.

Proof. As in the proof of Lemma 3.1, for $k \in K$ let $\phi_{k}$ be the homomorphism from $H$ into $A$ defined by $\phi_{k}(h)=\alpha(h, k)$. Clearly $K^{\perp} \subseteq\langle k\rangle^{\perp}=\operatorname{ker}\left(\phi_{k}\right)$, and thus we can think of $\phi_{k}$ as an element of $\operatorname{Hom}\left(H / K^{\perp}, A\right)$. Now define a map $\psi$ from $K$ into $\operatorname{Hom}\left(H / K^{\perp}, A\right)$ by $\psi(k)=\phi_{k}$. This map is clearly a homomorphism and its kernel is $H^{\perp}$. Thus $K / H^{\perp}$ is isomorphic to its image under this map. The proof for $H / K^{\perp}$ is identical.

So far we did not explicitly need any of the groups to be finite. However, from now on all groups considered, except $\mathbb{C}^{\times}$, are finite.

(3.3) Corollary. Suppose $\alpha$ is a bilinear pairing from $H \times K$ into an abelian group $A$. Then

(i) the prime $p$ divides $\left|K / H^{\perp}\right|$ if and only if it divides $\left|H / K^{\perp}\right|$,

(ii) if the prime $p$ divides $\left|H / K^{\perp}\right|$ or $\left|K / H^{\perp}\right|$ then it divides $|A|$,

(iii) the groups $H / K^{\perp}$ and $K / H^{\perp}$ have equal exponents, and their exponent is no greater than the exponent of $A$.

Proof. If $L$ and $A$ are finite abelian groups, write $L=L_{1} \times L_{2} \times$ : $\cdots \times L_{n}$ and $A=A_{1} \times \cdots \times A_{m}$ where $L_{i}$ and $A_{j}$ are cyclic. In this case it is well known that $\operatorname{Hom}(L, A)$ is isomorphic to a direct product of $n m$ cyclic groups $D_{i j}$ where the order of $D_{i j}$ is the greatest common divisor of $\left|L_{i}\right|$ and $\left|A_{j}\right|$. Now all the conclusions follow from this together with Proposition 3.2. 
We will now focus our attention to the case where we have a bilinear pairing $\alpha$ from $H \times K$ into a finite cyclic group $C$. By composing $\alpha$ with a faithful linear character of $C$, we get a bilinear pairing into the multiplicative group of nonzero complexes $\mathbb{C}^{\times}$. Since $H^{\perp}$ and $K^{\perp}$ remain the same after this change of perspective, without loss of generality we can assume $\alpha$ to be a bilinear pairing into $\mathbb{C}^{\times}$.

(3.4) Corollary. Let $\alpha: H \times K \rightarrow \mathbb{C}^{\times}$be a bilinear pairing. Then $H / K^{\perp}$ is abelian and isomorphic to $K / H^{\perp}$. In particular, assume $|H|=|K|$. Then $\alpha$ is left (or right) nondegenerate if and only if $\alpha$ is nondegenerate.

Proof. Both $H / K^{\perp}$ and $K / H^{\perp}$ are abelian by Lemma 3.1, and therefore $K / H^{\perp}$ is isomorphic to $\operatorname{Hom}\left(K / H^{\perp}, \mathbb{C}^{\times}\right)$. By Proposition 3.2 we have $H / K^{\perp} \cong \operatorname{Hom}\left(K / H^{\perp}, \mathbb{C}^{\times}\right) \cong K / H^{\perp}$. Similarly $K / H^{\perp} \cong$ $H / K^{\perp}$. Thus it follows that $H / K^{\perp}$ is isomorphic to $K / H^{\perp}$.

We say that $\alpha$ is a bilinear form on $G$ if $\alpha$ is a bilinear pairing from $G \times G$ into $\mathbb{C}^{\times}$. A bilinear form $\alpha$ on $G$ is said to be alternating if $\alpha(g, g)=1$ for all $g \in G$.

The following is immediate from Lemma 3.1.

(3.5) Corollary. Let $\alpha$ be a nondegenerate bilinear form on $G$. Then $G$ is abelian.

We can prove the following useful lemma.

(3.6) Lemma. Let $\alpha$ be a nondegenerate bilinear form on $G$. Then for any $c \in G$ there exists $d \in G$ such that $o(c)=o(\alpha(c, d)) \leq o(d)$.

Proof. For $g \in G$ let $\phi_{g}$ be the homomorphism of $G$ into $\mathbb{C}^{\times}$ defined by $\phi_{g}(x)=\alpha(g, x)$. Now define $\psi: G \rightarrow \operatorname{Irr}(G)$ by $\psi(g)=$ $\phi_{g}$. It is clear that $\psi$ is a homomorphism and its kernel is $G^{\perp}$. However $\alpha$ is nondegenerate and thus $\psi$ is one to one. It follows that $\psi$ is an isomorphism. Therefore if $o(c)$ is $m$ then so is $o\left(\phi_{c}\right)$. Now image of $\phi_{c}$ in $\mathbb{C}^{\times}$is contained in the group of $n$th roots of unity where $n$ is the exponent of $G$. Thus image of $\phi_{c}$ is cyclic. Let $d$ be an element of $G$ such that $\alpha(c, d)$ generates the image of $\phi_{c}$. Now $\alpha(c, d)^{m}=\alpha\left(c^{m}, d\right)=1$, and if $\alpha(c, d)^{k}=1$ then $m=o\left(\phi_{c}\right) \leq k$. Thus the order of $\alpha(c, d)$ is $m$, which in turn implies that the order of $d$ in $G$ cannot be smaller than $m$. The proof is complete. 
The following well-known theorem follows with a little effort from Lemma 3.6, and induction.

(3.7) THEOREM. Let $\alpha$ be a nondegenerate alternating bilinear form on a group $G$. Then $G \cong H \times H$ where $H$ is abelian.

Proof. See Proposition 3 of [2].

If $\alpha$ is a bilinear form on $G$, then clearly $\alpha \in Z\left(G, \mathbb{C}^{\times}\right)$. We would like to investigate the converse relationship, and use that to get a necessary condition for a group to be a ctfg.

(3.8) Theorem. Let $\alpha \in Z\left(G, \mathbb{C}^{\times}\right)$, and let $H$ and $K$ be subgroups of $G$ with $[H, K]=1$. Define $f_{\alpha}: H \times K \rightarrow \mathbb{C}^{\times}$by $f_{\alpha}(h, k)=$ $\alpha(h, k) \alpha(k, h)^{-1}$. Then $f_{\alpha}$ is a bilinear pairing from $H \times K$ into $\mathbb{C}^{\times}$, and $f_{\alpha}=f_{\beta}$ if $[\alpha]=[\beta]$. In particular for any $x \in G$ we have that $f_{\alpha}$ is a bilinear pairing from $\langle x\rangle \times \mathbf{C}_{G}(x)$ into $\mathbb{C}^{\times}$.

Proof. This is essentially the calculation on page 132 of [11].

(3.9) Corollary. Let $\alpha \in Z\left(G, \mathbb{C}^{\times}\right)$, and let $f_{\alpha}$ be as above. Then for $x \in G$, we have $f_{\alpha}$ is the trivial map on $\langle x\rangle \times \mathbf{C}_{G}(x)$ if and only if $x$ is $\alpha$-special. In particular, if $G$ is abelian and $\mathbb{C}^{\alpha}[G]$ simple then $f_{\alpha}$ is a nondegenerate alternating bilinear form on $G$.

Proof. The first assertion is immediate from 3.8 and the definition of $\alpha$-special. The second follows from the first and Lemma 2.3.

(3.10) Corollary. $G$ is an abelian ctfg if and only if $G \cong H \times H$ with $H$ abelian.

Proof. One direction follows from Corollary 3.9 together with Theorem 2.7. The other direction follows from a straightforward construction (see Lemma 2 of [3]).

(3.11) Proposition. Assume $G$ is a ctfg. Let $x$ be a nonidentity element of $G$, and $J$ any set such that $\mathbf{C}_{G}(x)=\left\langle J, \mathbf{C}_{G}(x)^{\prime}\right\rangle$. Then $x \notin \bigcap\langle y\rangle$ where the intersection is taken over all $y \in J$.

Proof. Assume the contrary for $x \in G$. Since $G$ is a ctfg, by Theorem 2.7 and Lemma 2.3 we get a 2-cocycle $\alpha$ such that there are no nonidentity $\alpha$-special elements. However we claim that $x$ is $\alpha$-special. It follows from Corollary 3.9 that we need to show that $f_{\alpha}$ 
is trivial. Now if $y \in J$ then by the hypothesis we have $x=y^{i}$, and thus $f_{\alpha}(x, y)=f_{\alpha}(y, y)^{i}=1$. Therefore $J$ is contained in $\langle x\rangle^{\perp}$. On the other hand by Lemma 3.1 we have that the commutator subgroup of $\mathbf{C}_{G}(x)$, that is, $\mathbf{C}_{G}(x)^{\prime}$, is also contained in $\langle x\rangle^{\perp}$. It follows that $\mathbf{C}_{G}(x) \subseteq\langle x\rangle^{\perp}$. Thus $f_{\alpha}:\langle x\rangle \times \mathbf{C}_{G}(x) \rightarrow \mathbb{C}^{\times}$is trivial, and we have the required contradiction.

(3.12) Corollary (S. Gagola). Let $G$ be a nontrivial ctfg. Then $G$ contains no selfcentralizing cyclic subgroups.

4. Local characterization of ctfgs. In this section we will obtain a local characterization for a group to be a ctfg. Our starting point is a theorem of DeMeyer and Janusz. However later we need a generalization of one of its directions, which we will present first. (The $\mathrm{H}^{+}$ notation was introduced after Lemma 2.3.)

(4.1) Proposition. Let $(\Gamma, N)$ be a central extension by $G$, and let $H$ be a subgroup of $G$. Assume that $N$ is fully ramified in $H^{+}$. Then there exists $\alpha \in Z\left(G, \mathbb{C}^{\times}\right)$with $\mathbb{C}^{\beta}[H]$ simple where $\beta=\alpha_{H}$.

Proof. This follows directly from Proposition 2.4. Let $\alpha$ be defined as in the statement of that Proposition where $\lambda$ is chosen to be the linear character of $N$ that is fully ramified in $H^{+}$. Then $\beta=\alpha_{H}$ is the corresponding cocycle for $\left(H^{+}, N\right)$ which is a central extension by $H$. Now according to Proposition 2.4 the number of $\beta$-special classes of $H$ is the same as $\left|\operatorname{Irr}\left(H^{+} \mid \lambda\right)\right|$. This number is one since $\lambda$ is fully ramified in $H^{+}$. Thus by Lemma 2.3 we have that $\mathbb{C}^{\beta}[H]$ is simple.

(4.2) Proposition. Let $(\Gamma, \mathbf{Z}(\Gamma))$ be a central extension by $G$, where $\Gamma$ is of central type. Then $\mathbf{Z}(\Gamma)$ is fully ramified in $\mathrm{H}^{+}$, where $H$ is a Hall $\pi$-subgroup of $G$ for any set of primes $\pi$.

Proof. This is immediate from Proposition 2.9 of [14].

(4.3) Theorem. (DeMeyer and Janusz). Let $\alpha \in Z\left(G, \mathbb{C}^{\times}\right)$. Then $\mathbb{C}^{\alpha}[G]$ is simple if and only if $\mathbb{C}^{\beta}[P]$ is simple for all Sylow p-subgroups $P$ of $G$, where $\beta=\alpha_{P}$.

Proof. This is Corollary 4 of [3]. One direction follows from Propositions 4.1 and 4.2. 
Note that this is not yet a local characterization. To check whether or not $G$ is a ctfg we need the existence of a 2-cocycle of $G$ with certain properties.

(4.4) LEMMA. Let $G$ be a finite group. Suppose that for each prime divisor $p_{i}$ of $|G|$ we have $P_{i}$ a Sylow $p_{i}$-subgroup of $G$. Let $\alpha_{i} \in$ $H\left(P_{i}, \mathbb{C}^{\times}\right)$and assume that $\alpha_{i}$ is stable for each $i$. Then there exists $\alpha \in H\left(G, \mathbb{C}^{\times}\right)$with $\alpha_{P_{1}}=\alpha_{i}$ for all $i$. In particular, $\mathbb{C}^{\alpha_{i}}\left[P_{i}\right]$ is isomorphic to $\mathbb{C}^{\beta}\left[P_{i}\right]$ for each $i$ where $\beta=\alpha_{P_{1}}$

Proof. By Proposition 2.1(iv) we have that $H\left(P_{i}, \mathbb{C}^{\times}\right)$is a $p_{i}$-group. Since $\left|G: P_{i}\right|$ is a number relatively prime to $p_{i}$, we can find integers $u_{i}$ and $v_{i}$ such that $u_{i}\left|G: P_{i}\right|+v_{i}\left|H\left(P_{i}, \mathbb{C}^{\times}\right)\right|=1$. Let $\alpha=\sum_{i} u_{i} \alpha_{i}^{G}$. Note that by Proposition 2.1(ii) we have $\left(\alpha_{i}^{G}\right)_{P_{l}}=\left|G: P_{i}\right| \alpha_{l}$, and it follows from (iii) of the same proposition that $\left(\alpha_{i}^{G}\right)_{P_{j}}=0$ for $j \neq i$. Putting these together we get

$$
\alpha_{P_{l}}=u_{i}\left|G: P_{i}\right| \alpha_{i}=\left(1-v_{i}\left|H\left(P_{i}, \mathbb{C}^{\times}\right)\right|\right) \alpha_{i}=\alpha_{i} .
$$

(4.5) Theorem. A group $G$ is a ctfg if and only if every Sylow $p$ subgroup $P$ of $G$ has a 2-cocycle $\alpha$ such that $\mathbb{C}^{\alpha}[P]$ is simple and $[\alpha]$ is stable in $G$.

Proof. Assume that $G$ is a ctfg. The conclusion follows from Theorem 4.3, and Proposition 2.1(i). The latter stated that restrictions are stable. The converse is also immediate from Lemma 4.4 and Theorem 4.3.

Theorem 4.5 is closer to a local characterization of ctfgs. However the requirement that $[\alpha]$ has to be stable in $G$ is not a local property. Thus we are interested in a local characterization of stability. Let $H \subseteq K \subseteq G$. Assume that if for some $g \in G$ we have $y_{l}=x_{i}^{g}$, for $\left\{x_{i}\right\}$ and $\left\{y_{i}\right\}$ two arbitrary subsets of $H$, then we can find $k \in K$ such that $y_{i}=x_{i}^{k}$ for all $i$. Then we say that $K$ controls $G$-fusion of sets in $H$. Note that if $P$ is a Sylow $p$-subgroup of $G$, then $\mathbf{N}_{G}(P)$ controls $G$-fusion of sets in $\mathbf{C}_{G}(P)$.

(4.6) Lemma. Let $H \subseteq K \subseteq G$. Assume that $K$ controls $G$-fusion of sets in $H$. Suppose $\alpha \in Z\left(H, \mathbb{C}^{\times}\right)$, and $[\alpha]$ is stable in $K$. Then $[\alpha]$ is stable in $G$.

Proof. Let $g$ be an arbitrary element of $G$. We need to show that $[\alpha]$ and $\left[\alpha^{g}\right]$ are the same when restricted to $H \cap H^{g}$. Now $K$ 
controls $G$-fusion of sets in $H$. Pick the set to be all of $H \cap H^{g}$. Thus there exits $k \in K$ such that we have $u=u_{1}^{k}$ for every $u \in H \cap H^{g}$ with $u=u_{1}^{g}$, where $u_{1} \in H$. Let $v$ be another element of $H \cap H^{g}$ with $v_{1}$ defined similarly. Thus we have $\alpha^{g}(u, v)=\alpha\left(u_{1}, v_{1}\right)=$ $\alpha^{k}(u, v)$. Therefore $\left[\alpha^{g}\right]$ and $\left[\alpha^{k}\right]$ are the same when restricted to $H \cap H^{g}$ which is a subgroup of $H \cap H^{k}$. However, by hypothesis the restrictions of $[\alpha]$ and $\left[\alpha^{k}\right]$ to $H \cap H^{k}$ are equal. It follows that $[\alpha]$ and $\left[\alpha^{g}\right]$ are the same when restricted to $H \cap H^{g}$, and thus $[\alpha]$ is stable in $G$.

We remark that the proof of Lemma 4.6 also proves a similar statement about characters: If $H \subseteq K \subseteq G$ and $K$ controls $G$-fusion in $H$, then any irreducible character of $H$ that is stable in $K$, is also stable in $G$.

It is clear from Lemma 4.6 and Theorem 4.5 that results about controlling fusion can be used to find local characterizations of ctfgs. For example Alperin's fusion theorem asserts that fusion can be determined by local properties. Thus to decide whether the equivalence class of a given cocycle is stable in $G$, we only need information about the local structure, that is, the normalizers of nonidentity $p$ subgroups, of $G$. To make this precise we have to recall Alperin's theorem. If $P$ and $Q$ are Sylow $p$-subgroups of $G$, we shall say that the intersection $P \cap Q$ is tame provided $\mathbf{N}_{P}(P \cap Q)$ and $\mathbf{N}_{Q}(P \cap Q)$ are each Sylow $p$-subgroups of $\mathbf{N}_{G}(P \cap Q)$.

(4.7) Theorem (Alperin). Let $A$ and $B$ be two subsets of a Sylow p-subgroup $P$ of $G$ and suppose that $A^{x}=B$. Then there exist elements $x_{i}$ and Sylow p-subgroups $Q_{i}$ of $G$, for $1 \leq i \leq n$, and an element $y$ of $\mathbf{N}_{G}(P)$ which satisfy the following conditions:

(i) $x=x_{1} x_{2} \cdots x_{n} y$.

(ii) $P \cap Q_{i}$ is a tame intersection for $1 \leq i \leq n$.

(iii) $x_{i}$ is a p-element of $\mathbf{N}_{G}\left(P \cap Q_{i}\right)$, for $1 \leq i \leq n$.

(iv) $A \subseteq P \cap Q_{1}$, while $A^{x_{1} x_{2} \cdots x_{i}} \subseteq P \cap Q_{i+1}$ for $1 \leq i \leq n-1$.

Proof. This is Theorem 2.6 of Chapter 7 of [5].

(4.8) TheOREM. A group $G$ is a ctfg if and only if every Sylow $p$ subgroup $P$ of $G$ has a 2-cocycle $\alpha$ such that $\mathbb{C}^{\alpha}[P]$ is simple and $[\alpha]$ is stabilized by $\mathbf{N}_{G}(P \cap Q)$ for all Sylow p-subgroups $Q$ for which $P \cap Q$ is tame and nontrivial. 
Proof. By Theorem 4.5 we need to show that $[\alpha]$ is stable in $G$. Let $x \in G$, and let $B=P \cap P^{x}$. We would like to show that $\left[\alpha^{x}\right]_{B}=[\alpha]_{B}$. Let $A=B^{x^{-1}}$, then apply Theorem 4.7 and get $x=x_{1} x_{2} \cdots x_{n} y$ with the appropriate properties.

Claim. $[\alpha]$ and $\left[\alpha^{x_{2} x_{2} \cdots x_{l}}\right]$ are equal when restricted to $A^{x_{1} x_{2} \cdots x_{i}}$.

Proof of Claim. We induct on $i$. For $i=1$, we have that $x_{1}$ is an element of $\mathbf{N}_{G}\left(P \cap Q_{1}\right)$ which stabilizes $[\alpha]$. The claim follows since $A^{x_{1}} \subseteq P \cap P^{x_{1}}$. Now assume the result for $i=k-1$. It immediately follows that the restrictions of $\left[\alpha^{x_{k}}\right]$ and $\left[\alpha^{x_{1} x_{2} \cdots x_{k}}\right]$ to $A^{x_{1} x_{2} \cdots x_{k}}$ are equal. However $x_{k}$ is an element of $\mathbf{N}_{G}\left(P \cap Q_{k}\right)$ which stabilizes $[\alpha]$. Thus $[\alpha]$ and $\left[\alpha^{x_{k}}\right]$ are equal when restricted to $A^{x_{1} x_{2} \cdots x_{k}}$ since this last group is contained in $P \cap P^{x_{k}}$. The claim now follows.

Applying an identical argument to the conclusion of the claim for $i=n$, we get $[\alpha]_{B}=\left[\alpha^{y}\right]_{B}=\left[\alpha^{x}\right]_{B}$ since $B \subseteq P \cap P^{y}$. The proof is complete.

Theorem 4.8 is a local characterization of ctfgs, and Theorem A is just a less technical corollary of it. It should be noted that in general this result is hard to use; however in some special cases it can be useful. This will be demonstrated in the next section when we will turn our attention to groups with abelian Sylow subgroups.

5. Ctfgs with abelian Sylows. In this section we will apply the results of the previous section to obtain a necessary and sufficient condition for an odd group with abelian Sylow subgroups to be a ctfg. The first proposition is stated more generally than is needed here. However the more general setting will be used in the subsequent sections.

Let $H$ be a subgroup of $G$ and let $f$ be a bilinear form on $H$. We say that $f$ is preserved by (or invariant in) $K$ a subgroup of $G$ if $K \subseteq \mathbf{N}_{G}(H)$ and $f\left(x^{k}, y^{k}\right)=f(x, y)$ for all $k \in K$.

(5.1) Proposition. Let $(\Gamma, N)$ be a central extension by $G$, and let $H$ be an abelian subgroup of $G$. Assume that $N$ is fully ramified in $H^{+}$. Then there exists a nondegenerate alternating bilinear form on $H$ that is preserved by $\mathbf{N}_{G}(H)$.

Proof. Proposition 4.1 applies and we get $\alpha \in Z\left(G, \mathbb{C}^{\times}\right)$such that $\mathbb{C}^{\beta}[H]$ is simple, where $\beta$ is the restriction of $\alpha$ to $H$. Now define $f(x, y)=\beta(x, y) \beta(y, x)^{-1}$. By Theorem 3.8 we have that $f$ is a bilinear form on $H$. To show $f$ is nondegenerate we need to show 
that $H^{\perp}$ is the trivial group. If $x \in H^{\perp}$ then $\beta(x, y)=\beta(y, x)$ for all $y \in H$, and thus $x$ is a $\beta$-special element of $H$. However $\mathbb{C}^{\beta}[H]$ is simple and therefore the identity is the only $\beta$-special element of $H$ by Lemma 2.3. This implies $H^{\perp}=1$ as needed. Clearly $f$ is alternating. It remains to show that $f$ is preserved by $\mathbf{N}_{G}(H)$. Now $\beta$ is the restriction of $\alpha$ to $H$, and by Lemma 2.1(i) restrictions are stable. Thus $[\beta]$ is stable in $G$. Therefore for $n \in \mathbf{N}_{G}(H)$ we have $\left[\beta^{n^{-1}}\right]=[\beta]$. Now according to Theorem 3.8 equivalent cocycles give identical bilinear pairings and thus:

$$
\begin{aligned}
f\left(x^{n}, y^{n}\right) & =\beta\left(x^{n}, y^{n}\right) \beta\left(y^{n}, x^{n}\right)^{-1}=\beta^{n^{-1}}(x, y) \beta^{n^{-1}}(y, x)^{-1} \\
& =\beta(x, y) \beta(y, x)^{-1}=f(x, y) .
\end{aligned}
$$

We remark here that the preceding theorem puts somewhat surprising restrictions on some configurations. To see an example of this, which will be used later, we make the following definition: Let $H$ be an elementary abelian subgroup of $G$ of order $p^{n}$. Now $H$ can be thought of as a vector space of dimension $n$ over the field with $p$ elements. The automorphism group of $H$ can now be identified with $\mathrm{GL}(n, p)$. Thus the action of every element $\gamma$ of $\mathbf{N}_{G}(H) / \mathbf{C}_{G}(H)$ on $H$ is the same as the action of the corresponding matrix linear transformation on the vector space. If the latter matrix is in $\operatorname{SL}(n, p)$ we say that $\gamma$ acts with determinant one.

Now if the abelian subgroup $H$ in the theorem is an elementary abelian $p$-group (of necessarily even rank by Theorem 3.7), then by virtue of preserving a nondegenerate alternating bilinear form on $H$, we have that $\mathbf{N}_{G}(H) / \mathbf{C}_{G}(H)$ acts on $H$ as a subgroup of $\operatorname{Sp}(f)$, the symplectic group. This group in turn is a subgroup of the special linear group $\operatorname{SL}(n, p)$, where $n$ is the rank of $H$. (See p. 336 of [15] for the definition and facts about $\operatorname{Sp}(f)$.) Thus $\mathbf{N}_{G}(H) / \mathbf{C}_{G}(H)$ must act on $H$ with determinant one. As an example, consider $G=S_{3} \times S_{3}$, the normalizer mod centralizer of the Sylow 3-subgroup $P$ does not act on $P$ with determinant one. Thus the curious fact follows that there is no $(\Gamma, N)$ a central extension by $G$ such that $N$ is fully ramified in $P^{+}$. The following corollary records the part of the above argument that we will need later.

(5.2) Corollary. Let $(\Gamma, N)$ be a central extension by $G$, and let $H$ be an elementary abelian p-subgroup of $G$. Assume that $N$ is fully ramified in $H^{+}$. Then every element of $\mathbf{N}_{G}(H) / \mathbf{C}_{G}(H)$ acts on $H$ with determinant one. 
For the rest of this section we will be looking for a converse to the following Corollary of 5.1:

(5.3) Corollary. Let $G$ be a ctfg with an abelian Sylow p-subgroup $P$. Then $P$ admits a nondegenerate alternating bilinear form that is preserved by $\mathbf{N}_{G}(P)$.

Proof. This is immediate from Proposition 5.1 and Proposition 4.2 .

We want to use Theorem 4.5 in the case where the Sylow subgroups are abelian. First we need the following lemma on stability.

(5.4) LEMMA. Let $P$ be an abelian Sylow p-subgroup of a group $G$. Assume that $\alpha \in H\left(P, \mathbb{C}^{\times}\right)$is stable in $\mathbf{N}_{G}(P)$. Then $\alpha$ is stable in $G$.

Proof. Since $P$ is abelian, it is contained in the $\mathrm{C}_{G}(P)$. Furthermore $\mathbf{N}_{G}(P)$ controls $G$-fusion of sets in $\mathbf{C}_{G}(P)$. The result is immediate from Lemma 4.6.

(5.5) Theorem. Let $G$ be a group with abelian Sylow subgroups. Assume that for every Sylow subgroup $P$ of $G$ there exists $\alpha \in$ $Z\left(P, \mathbb{C}^{\times}\right)$such that $\mathbb{C}^{\alpha}[P]$ is simple and $[\alpha]$ is stable in $\mathbf{N}_{G}(P)$. Then $G$ is a ctfg.

Proof. This is immediate from Theorem 4.5 and Lemma 5.4.

We can make the above theorem easier to use by substituting appropriate bilinear maps for cocycles. The following lemmas about abelian groups allow us to do just that. Our main result in this direction, that is, Theorem 5.9, is a generalization of a result of DeMeyer and Janusz (Theorem 6 of [3]) where they consider the case where each abelian Sylow subgroup is elementary abelian of rank two. We have included Lemma 5.7 to point out a fact that was overlooked in that proof.

(5.6) Lemma. Let $P$ be an odd group. Assume that $\alpha: P \times P \rightarrow \mathbb{C}^{\times}$ is a nondegenerate alternating bilinear form on $P$. Then $\mathbb{C}^{\alpha}[P]$ is simple.

Proof. First of all note that $P$ is abelian by Corollary 3.5. Also $\alpha$ is alternating which implies that $\alpha(g, h)=\alpha(h, g)^{-1}$ for all elements $g$ and $h$ of $P$ (just look at $1=\alpha(g h, g h)$ ). By Lemma 2.3 we will be done as soon as we show that the identity is the only $\alpha$-special element 
of $P$. Assume $g \in P$ is $\alpha$-special. Since $P$ is abelian this means that for all $x \in P$ we have $\alpha(g, x)=\alpha(x, g)=\alpha(g, x)^{-1}$. It follows that $o(\alpha(g, x))$ divides two for all $x \in P$. However $o(\alpha(g, x))$ is odd since $\alpha(\cdot, x): P \rightarrow \mathbb{C}^{\times}$is a homomorphism. Thus $\alpha(g, x)=1$ for all $x \in P$ which implies that $g$ is contained in $P^{\perp}$. This completes the proof since $\alpha$ is nondegenerate which means that $g$ is the identity element.

The above certainly does not work for even groups.

(5.7) Lemma. Let $P$ be an even group. Assume that $\alpha$ is an alternating bilinear form on $P$. Then $\mathbb{C}^{\alpha}[P]$ cannot be simple.

Proof. Let $x$ be an element of order two in $P$. For all $g \in P$ it follows that $\alpha(x, g)^{2}=\alpha\left(x^{2}, g\right)=1$. Thus $\alpha(x, g)=\alpha(x, g)^{-1}=$ $\alpha(g, x)$ for all $g \in P$, where the last equality follows since $\alpha$ is alternating. However this means that $x$ is $\alpha$-special which implies by Lemma 2.3 that $\mathbb{C}^{\alpha}[P]$ is not simple.

However, we can remedy the situation at least for the Klein four group.

(5.8) LemMA. Let $P$ be the Klein four group. Then there exists a nondegenerate bilinear form $\alpha: P \times P \rightarrow \mathbb{C}^{\times}$such that $\mathbb{C}^{\alpha}[P]$ is simple, and with the additional property that if $P$ is a Sylow 2-subgroup of a group $G$ then $[\alpha]$ is preserved by $\mathbf{N}_{G}(P)$.

Proof. Let $x$ and $y$ be two generators of $P$. Define $\alpha$ as follows:

$$
\begin{aligned}
& \alpha(g, g)=-1 \text { for all } 1 \neq g \in P, \\
& \alpha(g, h)=-\alpha(h, g) \text { for all nonidentity elements } \\
& \qquad g, h \in P \text { with } g \neq h, \\
& \alpha(x, y)=1,
\end{aligned}
$$

and extend linearly to other elements. Note that by the previous lemma $\alpha$ could not have been alternating. The twisted group algebra is simple since clearly $\alpha$ does not have any nonidentity $\alpha$-special elements. Now assume that $P$ is a Sylow 2-subgroup of a group $G$. Clearly $\alpha$ is preserved by the centralizer of $P$, and $\mathbf{N}_{G}(P) / \mathbf{C}_{G}(P)$ will be isomorphic to a subgroup of a Sylow 3-subgroup of $\operatorname{GL}(2,2)$. It can easily be checked that the elements of order 3 in $\operatorname{GL}(2,2)$ preserve $\alpha$. 
Putting together Theorem 5.5 and Lemmas 5.6 and 5.8 we get Theorem B:

(5.9) Theorem. Assume $G$ is a group such that $16+|G|$. Then $G$ is a ctfg with abelian Sylow subgroups if and only if all odd Sylow subgroups have a nondegenerate alternating bilinear form that is preserved by their normalizer, and the 2-Sylow subgroup is either trivial or the Klein four group.

6. Subnormal subgroups. The purpose of this section is to prove that the nonabelian group of order $p q$, where $p \mid q-1$ and $p$ and $q$ are primes, cannot be a subnormal subgroup of a ctfg. This result is interesting when contrasted to a result of Gagola's (Theorem 1.2 of [4]) which says that any solvable group is a subgroup of some ctfg.

To start with we need some facts about subnormal subgroups, and two results from character theory.

(6.1) Proposition. Let $A$ and $B$ be two subnormal subgroups of G. Then

(i) if $A \subseteq H \subseteq G$ then $A$ is subnormal in $H$,

(ii) if $N$ is a normal subgroup of $G$ then $A N / N$ is subnormal in $G / N$,

(iii) $A^{\infty} B=B A^{\infty}$,

(iv) if $A$ and $B$ are $\pi$-groups then so is $\langle A, B\rangle$.

Proof. (i) and (ii) are straightforward. For (iii) see [16]. We give a proof of (iv). Let $H=\langle A, B\rangle$. We will prove by induction on $|H|$ that $A^{H}$, the normal closure of $A$ in $H$, is a $\pi$-group. Let $D=A^{H}$; then $D$ is a proper subgroup of $H$ since $A$ is subnormal in $H$ by (i). By the inductive hypothesis $A^{D}$ is a $\pi$-group. Thus

$$
A^{D} \subseteq O_{\pi}(D) \operatorname{char} D \triangleleft H .
$$

However $D$ is the smallest normal subgroup of $H$ containing $A$. It follows that $O_{\pi}(D)=D$, and therefore $D$ is a $\pi$-group. Now $H=D B$ must also be a $\pi$-group.

(6.2) Lemma. Let $\mathbf{Z}(\Gamma) \subseteq K \triangleleft \Gamma$. Assume that $\lambda \in \operatorname{Irr}(\mathbf{Z}(\Gamma))$ is fully ramified in $\Gamma$, and choose $\phi \in \operatorname{Irr}(K \mid \lambda)$. Then

$$
t \phi(1)^{2}=|K: \mathbf{Z}(\Gamma)|,
$$

where $t=|\operatorname{Irr}(K \mid \lambda)|$ is the index of $I_{\Gamma}(\phi)$ in $\Gamma$. 
Proof. The proof is straightforward and the result is a special case of Corollary 2.5 of [14].

(6.3) Lemma. Let $A$ act on $G$ and let $N \triangleleft G$ be $A$-invariant. Assume that $(|A|,|G / N|)=1$. Let $\theta \in \operatorname{Irr}(N)$ be A-invariant. Then $\theta^{G}$ has an $A$-invariant irreducible constituent $\chi$. If $\mathbf{C}_{G / N}(A)=1$, then $\chi$ is unique.

Proof. This is Theorem 13.31 and Problem 13.10 of [10]. In this section we only use this result with the additional hypothesis that $G / N$ is abelian. This simpler case is Lemma 2.5 of [9]. Its proof is a straightforward application of Glauberman's Lemma.

Now we are ready to prove Theorem $C$, the main result of this section.

(6.4) THEOREM. Let $p$ and $q$ be primes such that $p$ divides $q-1$. Let $S$ be the nonabelian group of order $p q$. Assume that $S$ is a subnormal subgroup of $G$. Then $G$ is not a ctfg.

Proof. Assume that $G$ is a ctfg. Then $G$ is solvable by Theorem 2.8 , and the Hall $\{p, q\}$-subgroup of $G$ containing $S$ is a ctfg by Proposition 4.2. Thus it will be enough to assume that $G$ is a $\{p, q\}$ group.

Let $A$ be the normal closure $S^{G}$ of $S$ in $G$. By the definition of the normal closure, $A$ is normal in $G$ and $A=\left\langle S_{1}, S_{2}, \ldots, S_{m}\right\rangle$ where each $S_{i}$ is isomorphic to $S$ and subnormal in $G$. In the group $S_{i}$, let $Q_{i}$ and $P_{i}$ be the subgroups of order $q$ and of order $p$, respectively. In fact rearrange the ordering if necessary so that $\left\{Q_{1}, Q_{2}, \ldots, Q_{k}\right\}$ is the set of all distinct subgroups in $\left\{Q_{1}, Q_{2}, \ldots, Q_{m}\right\}$. Let $K=$ $Q_{1} Q_{2} \cdots Q_{k}$.

Claim $1 . K$ is an elementary abelian $q$-group.

Proof. We have that $Q_{i}=S_{i}^{\infty}$, and thus it follows from Proposition 6.1(iii) that $Q_{i} Q_{j}$ is an elementary abelian group of order $q^{2}$. Therefore each $Q_{i}$ centralizes every other one, which implies that $K$ is an elementary abelian $q$-group.

Claim 2. $K$ is normal in $A$.

Proof. To prove that $K$ is normal in $A$, we show that every $S_{j}$ normalizes $K$. This completes the proof since the $S_{j}$ 's generate $A$. 
Now by Proposition 6.1(iii) we have $Q_{i} S_{j}=S_{j} Q_{i}$, and thus $K S_{j}=$ $S_{j} K$. It follows that $K S_{j}$ is a group with $K \cap S_{j}=Q_{j}$. Now we have $\left|K S_{j}: K\right|=\left|S_{j}: K \cap S_{j}\right|=p$. Since $p$ is the smallest prime divisor of $\left|K S_{j}\right|$, it follows that $K$ is normal in $K S_{j}$ and the proof of the claim is complete.

Claim 3. $K$ is a Sylow $q$-subgroup of $A$, and thus $K$ is normal in $G$.

Proof. The factor group $A / K$ is generated by $\left\{S_{i} K / K \mid 1 \leq i \leq m\right\}$. These are all $p$-groups and they are subnormal in $A / K$ by Proposition 6.1(ii). Now Proposition 6.1(iv) applies and we get that $A / K$ is a $p$ group.

\section{Claim 4. Every $Q_{i}$ normalizes every $S_{j}$.}

Proof. This is obvious if $Q_{i}$ is contained in $S_{j}$. So assume that it is not. Let $H=Q_{l} S_{j}$. Now $H$ is a group of order $p q^{2}$ by Proposition 6.1(iii). Applying Proposition 6.1(i) we get that $S_{j}$ is a subnormal subgroup of index $q$ in $H$. However $q$ is a prime and thus $H$ normalizes $S_{j}$.

Claim 5. There exist elements of $\mathbf{N}_{G}(K) / \mathbf{C}_{G}(K)$ that do not act on $K$ with determinant one.

Proof. We will show that $P_{1} \mathbf{C}_{G}(K) / \mathbf{C}_{G}(K)$ does not act on $K$ with determinant one. Let $|K|=q^{n}$. Think of $K$ as a vector space of dimension $n$ over the field of $q$ elements. Let $\left\{x_{1}, x_{2}, \ldots, x_{n}\right\}$ be a basis for $K$. Without loss of generality we can assume that $\left\langle x_{i}\right\rangle=Q_{i}$. Let $P_{1}=\langle\alpha\rangle$. Now $P_{1}$ is isomorphic to $P_{1} \mathbf{C}_{G}(K) / \mathbf{C}_{G}(K)$ which is isomorphically embedded in the automorphism group of $K$. This gives a natural identification of $\alpha$ with $\tilde{\alpha}$ an element of $\operatorname{GL}(n, q)$, the automorphism group of $K$. Now the claim will be proved if we show that the determinant of $\tilde{\alpha}$ is not one. The $i$ th column of $\tilde{\alpha}$ is the coordinate vector of $x_{i}^{\alpha}$ in terms of the basis of $K$. Clearly $x_{1}^{\alpha}=x_{1}^{r}$, where $r \not \equiv 1 \bmod (q)$ since $x_{1}$ and $\alpha$ are both in $S_{1}$. On the other hand by Claim 4 every $x_{i}$ normalizes $S_{1}$, and thus $\left[x_{i}, \alpha\right]=\alpha^{u} x_{1}^{v}$ for: some integers $u$ and $v$. This implies that $x_{i}^{\alpha}=x_{i} \alpha^{u} x_{1}^{v}$. However this last element must be in $K$ which means that $\alpha^{u}=1$. Therefore $x_{i}^{\alpha}=$ $x_{i} x_{1}^{v}$. So $\tilde{\alpha}$ is a triangular matrix with diagonal entries consisting of $r$ and $n-1$ ones. It follows that $\operatorname{det}(\tilde{\alpha})=r \not \equiv 1 \bmod (q)$, and the proof of the claim is complete. 
Claim 6. Let $P$ be a $p$-Sylow subgroup of $A$. Then $\mathbf{C}_{K}(P)=1$.

Proof. We have the coprime action of $P$ on the abelian group $K$, and thus by Fitting's Theorem: $A=\mathrm{C}_{K}(P) \times[P, K] P$. Assume that the centralizer of $P$ in $K$ is not trivial. Then $A$ will have a nontrivial homomorphic image of order power of $q$. However this is impossible. To see this assume that such a homomorphism exists. The image of $S_{i}$ under this homomorphism is trivial, since $S_{i}$ does not have a factor group of order $q$. It follows that the image of $A$ is trivial, for $A$ is generated by $\left\{S_{i} \mid 1 \leq i \leq m\right\}$. The contradiction proves the claim.

We had assumed that $G$ is a $\mathrm{ctfg}$, so let $(\Gamma, \mathbf{Z}(\Gamma))$ be a central extension by $G$, with $\lambda \in \operatorname{Irr}(\mathbf{Z}(\Gamma))$ fully ramified in $\Gamma$.

Claim 7. Every element of $\operatorname{Irr}\left(K^{+} \mid \lambda\right)$ is $P^{+}$invariant.

Proof. Let $\phi \in \operatorname{Irr}\left(K^{+} \mid \lambda\right)$; then, since $K^{+}$is normal in $\Gamma$, it follows from Lemma 6.2 that $t \phi(1)^{2}=|K|$. Thus $t$ is a $q$-number. In particular $p$ does not divide $t=\left|\Gamma: I_{\Gamma}(\phi)\right|$, where $I_{\Gamma}(\phi)$ denotes the inertia group of $\phi$ in $\Gamma$. This implies that $A^{+}$is contained in the inertia group of $\phi$. This proves the claim since $P^{+}$is a subgroup of $A^{+}$.

Claim 8. $\lambda$ is fully ramified in $K^{+}$.

Proof. The claim follows from Lemma 6.3. To apply the lemma replace $A$ by $P^{+} / \mathbf{Z}(\Gamma)$, and $G$ by $K^{+}$. In addition let $N$ be $\mathbf{Z}(\Gamma)$, and $\theta$ be $\lambda$. Now by the conclusion of the lemma there is a unique $P^{+}$invariant constituent of $\lambda^{G}$. However by Claim 7 all irreducible characters of $K^{+}$lying over $\lambda$ are $P^{+}$invariant. This implies that $\lambda$ is fully ramified in $K^{+}$.

Claim 9. The final contradiction.

Proof. Corollary 5.2 readily applies to our situation and we get that every element of $\mathbf{N}_{G}(K) / \mathbf{C}_{G}(K)$ acts on $K$ with determinant one. However this is in direct contradiction with Claim 5.

7. Normal subgroups. As was mentioned earlier any solvable group can be a subgroup of a ctfg, and in the last section we saw some restrictions on the subnormal subgroups of a ctfg. In this section we want to explore some restrictions on the normal subgroups of a ctfg. 
The purpose is, of course, to be able to eliminate a specific group as a possible ctfg by using these restrictions. The first such restrictions on normal subgroups were found by Howlett and Isaacs [6]. They showed that the index of a system normalizer of a normal subgroup of a ctfg must be a square. In [13] it was shown that a generalized quaternion group of order $2^{n} \quad(n>2)$, a semidihedral group of order $2^{n} \quad(n>3)$, or an extraspecial group of order $p^{3}$ with exponent $p^{2}$ $(p>2)$, cannot be normal subgroups of ctfgs. Here we will first look at some general restrictions and then proceed to study some specific classes of groups.

To prove our first result we need the following lemma:

(7.1) Lemma (Gallagher). Let $N \triangleleft \Gamma$, and let $\theta \in \operatorname{Irr}(N)$ be extendible to $\Gamma$ (that is there exists $\chi \in \operatorname{Irr}(\Gamma)$ with $\chi_{N}=\theta$ ). Then the characters $\beta \chi$ for $\beta \in \operatorname{Irr}(\Gamma / N)$ are irreducible, distinct for distinct $\beta$, and are all of the irreducible constituents of $\theta^{\Gamma}$. In particular, $|\operatorname{Irr}(\Gamma \mid \theta)|=|\operatorname{Irr}(\Gamma / N)|$.

Proof. This is Corollary 6.17 of [10].

(7.2) Proposition. Let $\Gamma$ be a group of central type, and $\mathbf{Z}(\Gamma) \subseteq$ $S \triangleleft \Gamma$. Let $\lambda \in \operatorname{Irr}(\mathbf{Z}(\Gamma))$ be fully ramified in $\Gamma$. Assume $\lambda$ is extendible to $S$. Then $S / \mathbf{Z}(\Gamma)$ is abelian.

Proof. Let $\varphi \in \operatorname{Irr}(S \mid \lambda)$. By Lemma 6.2 we have $t \varphi(1)^{2}=|S: \mathbf{Z}(\Gamma)|$, where $t=|\operatorname{Irr}(S \mid \lambda)|$. Since $\lambda$ is extendible to $S, \varphi(1)=1$ and by Lemma $7.1 t=|\operatorname{Irr}(S / \mathbf{Z}(\Gamma))|$. Thus $|\operatorname{Irr}(S / \mathbf{Z}(\Gamma))|=|S / \mathbf{Z}(\Gamma)|$. So the number of conjugacy classes of $S / Z(\Gamma)$ is equal to its order. It follows that $S / \mathbf{Z}(\Gamma)$ is abelian.

We now get our first restriction on normal subgroups of ctfgs. This result will be used later.

(7.3) Corollary. Let $K$ be a noncyclic group with trivial multiplier. Assume that $K \triangleleft G$. Then $G$ is not a ctfg.

Proof. Note that if $K$ is abelian then, by Proposition 2.2(ii), it can have trivial multiplier only if it is cyclic. Thus $K$ is not abelian. Now assume that $G$ is a ctfg, and let $(\Gamma, \mathbf{Z}(\Gamma))$ be an extension by $G$ where $\lambda \in \operatorname{Irr}(\mathbf{Z}(\Gamma))$ is fully ramified in $\Gamma$. Now $K^{+} / \mathbf{Z}(\Gamma) \cong$ $K$ is not abelian, and so, by Proposition 7.2, the character $\lambda$ is not extendible to $K^{+}$. Proposition 2.4 now produces a nontrivial element of $H\left(K, \mathbb{C}^{\times}\right)=M(K)$. This contradiction completes the proof. 
(7.4) Lemma. Let the p-group $P$ act on $K$ and let $L \triangleleft K$ admit $P$ with $p \nmid|K: L|$ and $\mathbf{C}_{K}(P) \subseteq L$. Assume that $K$ is p-nilpotent. Then $\mathbf{C}_{K / L}(P)=1$.

Proof. This is Lemma 3.4 of [6].

In the proof of the following some of the ideas of Theorem 6.1 of [6] are used.

(7.5) THEOREM. Let $\Gamma$ be a group of central type with $\lambda \in \operatorname{Irr}(\mathbf{Z}(\Gamma))$ fully ramified in $\Gamma$. Let $\mathbf{Z}(\Gamma) \subseteq S \triangleleft \Gamma$. Assume that $S$ is not nilpotent. Then $\lambda$ cannot be extended to $\mathbf{F}(S)$, the Fitting subgroup of $S$.

Proof. Let $F=\mathrm{F}(S)<S$ and let $M / F$ be a chief factor of $\Gamma$ with $M \subseteq S$. Then $M / F$ is a $p$-group for some prime $p$ and we let $P \in \operatorname{Syl}_{p}(M)$. Let $H=\mathbf{N}_{\Gamma}(P)$ and observe that $P$ is not normal in $M$ since $F P=M$, and $M$ is not nilpotent. Thus $H<\Gamma$. By the Frattini argument $M H=\Gamma$. Of course $\mathbf{Z}(\Gamma) \subseteq H$ and $p \nmid|\Gamma: H|$. Let $K_{0}$ be the $p$-complement in $F$ and write $K=K_{0} \mathbf{Z}(\Gamma)$.

Let $\theta \in \operatorname{Irr}(K \mid \lambda)$. By Lemma 6.2 we have $t \theta(1)^{2}=|K: \mathbf{Z}(\Gamma)|$ where $t$ is the index of $I_{\Gamma}(\theta)$ in $\Gamma$. Now $|K: \mathbf{Z}(\Gamma)|=\left|K_{0}: K_{0} \cap \mathbf{Z}(\Gamma)\right|$ is a $p^{\prime}$ number. Thus $p \nmid t$. This forces $M \subseteq I_{\Gamma}(\theta)$ since $I_{\Gamma}(\theta)$ contains $K$ and $|M: K|$ is a power of $p$. Thus $M$ fixes all $\operatorname{Irr}(K \mid \lambda), P$ is a subgroup of $M$, and so all the characters in $\operatorname{Irr}(K \mid \lambda)$ are $P$-invariant.

Let $L=H \cap K$ then $L=\mathbf{Z}(\Gamma)\left(L \cap K_{0}\right)$. Now $L \cap K_{0}$ and $P$ are both normal in $H \cap M$ and their intersection is trivial. So $P \subseteq \mathbf{C}\left(L \cap K_{0}\right)$. This implies that $P \subseteq \mathbf{C}_{\Gamma}(L)$. So $\operatorname{Irr}(L \mid \lambda)$ are also $P$-invariant.

Now assume that $\lambda$ is extendible to $F$. By Proposition 7.2, the group $F / \mathbf{Z}(\Gamma)$ is abelian, and so $L$ is normal in $F$. Let $\varphi \in \operatorname{Irr}(L \mid \lambda)$. Since $\lambda$ extends to $F$ it must also extend to $L$ and $K$, and so $\varphi$ is linear and $\varphi_{\mathbf{Z}(\Gamma)}=\lambda$. In addition $\varphi$ is extendible to $K$ and so by Lemma 7.1 we have $|\operatorname{Irr}(L \mid \varphi)|=|\operatorname{Irr}(K / L)|=|K / L|$.

However, we can also apply the character correspondence in Lemma 6.3. We have that $P$ acts on $K$ and $L \triangleleft K$ is $P$-invariant. Also $(|P|,|K: L|)=1, K / L$ is abelian, and $\varphi \in \operatorname{Irr}(L)$ is $P$-invariant. Furthermore $\mathbf{C}_{K}(P) \subseteq \mathbf{N}_{\Gamma}(P) \cap K=L$, and so $\mathbf{C}_{K / L}(P)=1$ by Lemma 7.4. Thus by Lemma $6.3, \varphi^{K}$ has a unique $P$-invariant irreducible constituent. But we saw that all $\operatorname{Irr}(K \mid \lambda)$ are $P$-invariant, and so $\varphi^{K}$ must have a unique irreducible constituent. This contradicts the fact that $|\operatorname{Irr}(L \mid \varphi)|=|K: L|>1$, and completes the proof. 
Now, we are ready to prove Theorem D:

(7.6) Corollary. Assume that $G$ is a ctfg, and $S$ is a noncyclic normal subgroup of $G$. Then the Schur Multiplier of the Fitting group of $S, M(\mathbf{F}(S))$, is not trivial.

Proof. Note that if we knew that $\mathrm{F}(S)$ is not cyclic, then this result would be immediate from Corollary 7.3. Thus the additional content of this corollary is that $\mathbf{F}(S)$ cannot be cyclic.

We can assume that $S$ is not nilpotent, since if $S$ is nilpotent then $\mathbf{F}(S)=S$, and we are done by Corollary 7.3. Let $(\Gamma, \mathbf{Z}(\Gamma))$ be a central extension by $G$ with $\lambda \in \operatorname{Irr}(\mathbf{Z}(\Gamma))$ fully ramified in $\Gamma$. Now $\mathbf{F}\left(S^{+}\right) / \mathbf{Z}(\Gamma) \cong \mathbf{F}(S)$. By Theorem 7.5 , the character $\lambda$ cannot be extended to $\mathrm{F}\left(S^{+}\right)$, and thus by Proposition 2.4, the Schur multiplier of $\mathbf{F}\left(S^{+}\right) / \mathbf{Z}(\Gamma)$ is not trivial. This completes the proof.

Thus, for example, a generalized quaternion group or a group with all cyclic Sylow subgroups cannot be the Fitting subgroup of a noncyclic normal subgroup of a ctfg. In particular if the dihedral group of order 18 is a normal subgroup of $G$, then $G$ is not a ctfg. Note that the index of the system normalizer of $D_{18}$ is 9 , and thus $D_{18}$ could not be eliminated as a normal subgroup of a ctfg by the restriction on normal subgroups found in [6].

We now turn our attention to finding necessary and sufficient conditions for a certain class of groups (which include Frobenius groups) to be a normal subgroup of a ctfg. Some of the results of the previous sections will be used, but first a lemma is needed:

(7.7) Lemma. Let $H$ act on $N$, let $G=N \rtimes H$, then there is no $G$ fusion of sets in $H$. In particular, if $\alpha \in Z\left(H, \mathbb{C}^{\times}\right)$, then $[\alpha]$ is stable in $G$.

Proof. Let $x$ and $y$ be elements of $H$ with $x^{g}=y$. We can write $g=h n$ where $h \in H$ and $n \in N$. Now $u=x^{h}$ is an element of $H$. It follows that $y=u[u, n]$. Thus $[u, n] \in H \cap N=1$, which implies $y=u=x^{h}$. The generalization to sets is immediate, and the conclusion for cocycles follows from Lemma 4.6.

We now prove Theorem E:

(7.8) Theorem. Let $S$ be the semi-direct product $N \rtimes H$. Assume $(|N|,|H|)=1$, and $\mathbf{C}_{N}(H)=1$. Then $S$ is a normal subgroup of a 
ctfg if and only if

(a) $N$ has a 2-cocycle $\alpha$ with $\mathbb{C}^{\alpha}[N]$ simple (i.e. $N$ is a ctfg).

(b) $H$ is a normal subgroup of a ctfg $G_{0}$.

(c) $G_{0}$ acts on $N$ with $H$ acting as in $S$, and $\alpha$ admits $G_{0}$.

Proof. Assume $S \triangleleft G$, with $G$ a ctfg. Let $(\Gamma, \mathbf{Z}(\Gamma))$ be a central extension by $G$ where $\lambda \in \operatorname{Irr}(\mathbf{Z}(\Gamma))$ is fully ramified in $\Gamma$. Now $N$ is a normal Hall subgroup of $S$ and so it is normal in $G$.

Let $\varphi \in \operatorname{Irr}\left(N^{+} \mid \lambda\right)$. By Lemma 6.2, we have $t \varphi(1)^{2}=|N|$ where $t=\left|\Gamma: I_{\Gamma}(\varphi)\right|$. Thus $t$ divides $|N|$ and is relatively prime to $|H|$. This implies that $S^{+} \subseteq I_{\Gamma}(\varphi)$, i.e. $\varphi$ is invariant in $S^{+}$. Thus all irreducible characters of $N^{+}$lying over $\lambda$ are $H$-invariant. However, we can apply Lemma 6.3 , for the action of $H$ on $N^{+}$, and conclude that $\lambda^{N^{+}}$has a unique $H$-invariant constituent. It follows that $\left|\operatorname{Irr}\left(N^{+} \mid \lambda\right)\right|=1$, and thus $N^{+}$is fully ramified over $\mathbf{Z}(\Gamma)$. Thus $N$ is a ctfg.

Now we have to describe $G_{0}$, a ctfg, which will be a subgroup of $G_{1}=\mathbf{N}_{G}(H)$. First we claim that $G_{1} N=G$ and $G_{1} \cap S=H$. Let $g \in G$. By the Schur-Zassenhaus Theorem $H^{g}=H^{n}$ for some $n \in N$. This implies that $g n^{-1} \in G_{1}$, and thus $G_{1} N=G$. To prove $G_{1} \cap S=H$, let $K=G_{1} \cap S$. Since both $H$ and $K \cap N$ are normal in $K$, we have $K \cong H \times(K \cap N)$. Thus $K \cap N \subseteq \mathbf{C}_{N}(H)=1$, and so $K=H$. Note that as a consequence $G_{1} \cap N=1$. Now let $\pi$ be the set of prime divisors of $|H|$. Let $L / N \in \operatorname{Hall}_{\pi}(G / N)$, and $G_{0}=G_{1} \cap L$.

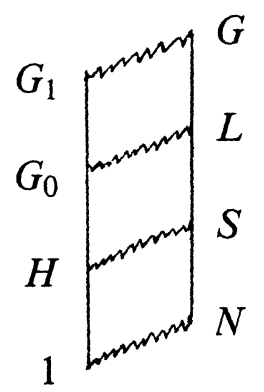

We claim that $G_{0}$ has the properties claimed in the statement of the theorem. We know that $\lambda$ is fully ramified in $\Gamma$, and we have proved that $\lambda$ is fully ramified in $N^{+}$, and thus $\varphi \in \operatorname{Irr}\left(N^{+} \mid \lambda\right)$ is fully ramified in $\Gamma$.

By Proposition 4.2, we conclude that $\varphi$ is fully ramified in $L^{+}$. Now $G_{0} \in \operatorname{Hall}_{\pi}(L)$, and so again by Proposition $4.2, \lambda$ is fully ramified in $G_{0}^{+}$. Thus $G_{0}$ is a ctfg with $H \triangleleft G_{0}$. Now $G_{0}$, as a subgroup of $L$, acts on $N$ with $H$ acting as in $S$. Since $\mathbf{Z}(\Gamma)$ is 
fully ramified in $N^{+}$, by Proposition 4.1 , there exists $\beta \in \mathbf{Z}\left(G, \mathbb{C}^{\times}\right)$ such that $\mathbb{C}^{\alpha}[N]$ is simple, where $\alpha=\beta_{N}$. By Lemma 2.1(i), $\alpha$ is stable in $G$, and thus it is invariant under the action of $G_{0}$.

To prove the converse, assume that $S$ is as given. We need to show that $S \triangleleft G$ with $G$ a ctfg. Without loss of generality, we can assume that $\left(|N|,\left|G_{0}\right|\right)=1$, since otherwise we can replace $G_{0}$ with an appropriate Hall subgroup. Let $G=N \rtimes G_{0}$. To prove $G$ is a ctfg we use Theorem 4.5. For every Sylow $p$-subgroup $P$ of $G$ we need a 2 -cocycle $\beta$ such that $\mathbb{C}^{\beta}[P]$ is simple, and $[\beta]$ is stable in $G$. For primes $p$ that divide $|N|$, let $\beta=\alpha_{p}$. Now $\mathbb{C}^{\beta}[P]$ is simple by Theorem 4.3, and $\beta$ is stable in $G$, since $\alpha$ is. For primes $p$ that divide $\left|G_{0}\right|$, let $\beta=\gamma_{p}$ where $\gamma$ is a cocycle of $G_{0}$ with $\mathbb{C}^{\gamma}\left[G_{0}\right]$ simple. Again $\mathbb{C}^{\beta}[P]$ is simple by Theorem 4.3 , and $[\beta]$ is stable in $G$ by Lemma 7.7. Thus the proof is complete by Theorem 4.5.

Let $(\Gamma, \mathbf{Z}(\Gamma))$ be a central extension by $G$, with $\lambda \in \operatorname{Irr}(\mathbf{Z}(\Gamma))$ fully ramified in $\Gamma$. Following Howlett and Isaacs [6], we say that $G$ is a reducible ctfg if $\lambda$ is fully ramified in some $N^{+} \triangleleft \Gamma$ with $\mathbf{Z}(\Gamma)<$ $N^{+}<\Gamma$. Otherwise $G$ is said to be irreducible. The following is an immediate Corollary of Theorem 7.8.

(7.9) Corollary. Let $G$ be an irreducible ctfg. Let $S \triangleleft G$. Then $S$ cannot be a Frobenius group.

We can also get a variation on Theorem 7.8, by relaxing the assumption that $\mathrm{C}_{N}(H)=1$. However, we then need to assume that $N$ is abelian.

(7.10) Theorem. Let $R$ be the semi-direct product $Q \rtimes P$. Assume that $Q$ is abelian, $(|Q|,|P|)=1$, and that $|Q|$ is odd. $R$ is a normal subgroup of a ctfg $G$ if and only if

(a) $[P, Q]$ has a nondegenerate alternating bilinear form $\alpha:[P, Q]$ $\times[P, Q] \rightarrow \mathbb{C}^{\times}$, i.e. $[P, Q]$ is a ctfg.

(b) $P$ is a normal subgroup of a ctfg, $H$.

(c) $H$ acts on $[P, Q]$ with $P$ acting as in $R$, and $\alpha$ is preserved by $H$.

Proof. Without loss of generality we can assume that $|H|$ and $|P|$ have the same prime divisors.

By Fitting's Theorem, $R=\mathrm{C}_{Q}(P) \times[P, Q] P$, and $[P, Q] P$ is a characteristic subgroup of $R$. This implies that $S=[P, Q] P$ is a normal subgroup of $G$. Let $N=[P, Q]$. Then $S=N \rtimes P$ with 
$\mathbf{C}_{N}(P)=1$. Now the conclusion follows from Theorem 7.8 and Corollary 3.9.

For the converse, again as in Theorem 7.8, we get that $S=[P, Q] P$ is a normal subgroup of $K=[P, Q] \rtimes H$ which is a ctfg. Now $R$ will be a normal subgroup of $K \times \mathrm{C}_{Q}(P) \times \mathrm{C}_{Q}(P)$ which is a ctfg.

8. Direct summands. We saw in $\S 3$ that any abelian group can be a direct summand of a ctfg. This is true because $H \times H$ is a ctfg for any abelian group $H$ (Corollary 3.10). Of course, because of the restrictions on the normal subgroups of a ctfg, not every solvable group can be a direct summand of a ctfg. In this section we get some new restrictions on direct summands of ctfgs.

We first need an easy lemma:

(8.1) LEMMA. Let $\lambda \in \operatorname{Irr}(\mathbf{Z}(\Gamma))$ be fully ramified in $\Gamma$ and suppose that $\mathbf{Z}(\Gamma) \subseteq N \triangleleft \Gamma$. Let $\varphi \in \operatorname{Irr}(N \mid \lambda)$. Then $\varphi$ is fully ramified in $I_{\Gamma}(\varphi)$.

Proof. This is Lemma 4.6 of [6]. The proof is easy.

(8.2) Proposition. Let $(\Gamma, \mathbf{Z}(\Gamma))$ be a central extension by $G$, and assume $\lambda \in \operatorname{Irr}(\mathbf{Z}(\Gamma))$ is fully ramified in $\Gamma$. Let $A \triangleleft G$. Assume that there is a subgroup $C$ of $G$ such that $A C=G$, and $\left[A^{+}, C^{+}, C^{+}\right]=1$. Then $G / A$ is ctfg by abelian.

Proof. Now $\left[C^{+}, A^{+}, C^{+}\right]=\left[A^{+}, C^{+}, C^{+}\right]=1$, and thus by the three subgroups lemma we have that $\left[C^{+}, C^{+}, A^{+}\right]$is trivial. Let $E^{+}=\mathbf{C}_{C^{+}}\left(A^{+}\right)$. It follows that $\left[C^{+}, C^{+}\right] \subseteq E^{+}$, and so $C^{+} / E^{+}$ is abelian. Now both $C^{+}$and $A^{+}$normalize $E^{+}$, and thus $E^{+}$is normal in $\Gamma$. As a consequence $A^{+} E^{+} \triangleleft \Gamma$, and $\Gamma / A^{+} E^{+}$is an abelian group. If $\varphi \in \operatorname{Irr}\left(A^{+} \mid \lambda\right)$, then $A^{+} E^{+} \subseteq I_{\Gamma}(\varphi)$. Thus $\Gamma / I_{\Gamma}(\varphi)$ is abelian, and $I_{\Gamma}(\varphi) / A^{+}$is a ctfg by Lemma 8.1 .

Theorem $\mathrm{F}$ follows immediately:

(8.3) Corollary. Assume $A$ is a direct summand of a ctfg. Then $A$ is ctfg by abelian.

It follows that the only dihedral groups that can be direct summands of ctfgs are the Klein four group, and the dihedral group of order eight. One can easily build examples to show that these are direct summands of ctfgs.

In conclusion, we mention some curious relations between system normalizers and ctfgs. Howlett and Isaacs [6] used one such fact in 
the proof of solvability of groups of central type. With a little effort, it follows from their work that if $G$ is a ctfg (a normal subgroup of a ctfg, a direct summand of a ctfg), then any system normalizer of $G$ is also a ctfg (a normal subgroup of a ctfg, a direct summand of a ctfg). This together with the results of previous sections and those of [13] puts severe restrictions on system normalizers of ctfgs (normal subgroups of ctfgs, direct summands of ctfgs).

\section{REFERENCES}

[1] A Babakhanian, Cohomological Methods in Group Theory, Marcel Dekker Inc., New York, 1972.

[2] F. DeMeyer, Galois theory in separable algebras over commutative rings, Illinois J. Math., 10 (1966), 287-295.

[3] F. DeMeyer and G. J. Janusz, Finite groups with an irreducible representation of large degree, Math. Z., 108 (1969), 145-153.

[4] S. M. Gagola, Characters fully ramified over a normal subgroup, Pacific J. Math., 55 (1974), 107-126.

[5] D. Gorenstein, Finite Groups, Chelsea Publishing Co., New York, 1980.

[6] R. B. Howlett and I. M. Isaacs, On groups of central type, Math. Z., 179 (1982), 555-569.

[7] B. Huppert, Endlichen Gruppen I, Springer Verlag, Berlin-Heidelberg-New York, 1967.

[8] I. M. Isaacs, On the character theory of fully ramified sections, Rocky Mountain J. Math., 13 (1983), 689-698.

[9] - Character correspondence in solvable groups, Advances in Math., 43 (1982), 284-306.

[10] Character Theory of Finite Groups, Academic Press, New York-San Francisco-London, 1976.

[11] N. Iwahori, and H. Matsumoto, Several remarks on projective representations of finite groups, J. Fac. Sci. Univ. Tokyo Sect. IA Math., 10 (1964), 129-146.

[12] R. A. Liebler and J. E. Yellen, In search of nonsolvable groups of central type, Pacific J. Math., 82 (1979), 485-492.

[13] S. Shahriari, On normal subgroups of capable groups, Arch. Math., 48 (1987), 193-198.

[14] __ Fully ramified subgroups, J. Algebra, 130 (1990), 198-218.

[15] M. Suzuki, Group Theory I, Springer Verlag, Berlin-Heidelberg-New York, 1982.

[16] H. Wielandt, Vertauschbare nachinvariante Untergruppen, Abh. Math. Sem. Univ. Hamburg, 21 (1957), 55-62.

Received December 15, 1988 and in revised form July 12, 1990. Research partially supported by the California State University, Northridge. 


\section{PACIFIC JOURNAL OF MATHEMATICS EDITORS}

\author{
V. S. VARADARAJAN \\ (Managing Editor) \\ University of California \\ Los Angeles, CA 90024-1555-05 \\ Herbert Clemens \\ University of Utah \\ Salt Lake City, UT 84112 \\ THOMAS ENRIGHT \\ University of California, San Diego \\ La Jolla, CA 92093
}

Nicholas ERCOLANI

University of Arizona

Tucson, AZ 85721

R. FINN

Stanford University

Stanford, CA 94305

VAUGHAN F. R. JONES

University of California

Berkeley, CA 94720

STEVEN KeRCKHOFF

Stanford University

Stanford, CA 94305
C. C. MOORE

University of California

Berkeley, CA 94720

Martin ScharlemanN

University of California

Santa Barbara, CA 93106

HAROLd STARK

University of California, San Diego

La Jolla, CA 92093

\section{ASSOCIATE EDITORS}
R. ARENS
E. F. BECKENBACH
B. H. NeUMANN
F. WolF
K. Yoshida (1906-1982)
(1904-1989)

SUPPORTING INSTITUTIONS

UNIVERSITY OF ARIZONA

UNIVERSITY OF BRITISH COLUMBIA

CALIFORNIA INSTITUTE OF TECHNOLOGY

UNIVERSITY OF CALIFORNIA

MONTANA STATE UNIVERSITY

UNIVERSITY OF NEVADA, RENO

NEW MEXICO STATE UNIVERSITY

OREGON STATE UNIVERSITY
UNIVERSITY OF OREGON

UNIVERSITY OF SOUTHERN CALIFORNIA

STANFORD UNIVERSITY

UNIVERSITY OF HAWAII

UNIVERSITY OF TOKYO

UNIVERSITY OF UTAH

WASHINGTON STATE UNIVERSITY

UNIVERSITY OF WASHINGTON 


\section{Pacific Journal of Mathematics}

\section{Vol. 151, No. $1 \quad$ November, 1991}

Fernanda Maria Botelho, Rotational entropy for annulus endomorphisms . . . 1

David F. Cowan, Some infinite chains in the lattice of varieties of inverse

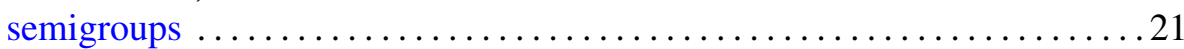

S. Greco and G. Raciti, The Lüroth semigroup of plane algebraic curves . . . 44

Kang-Tae Kim, Automorphism groups of certain domains in $\mathbf{C}^{n}$ with a

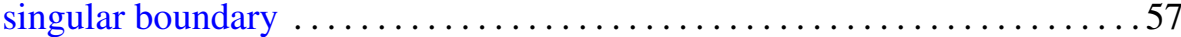

R. James Milgram, Surgery with finite fundamental group. I: The

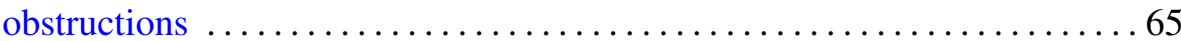

R. James Milgram, Surgery with finite fundamental group. II: The oozing

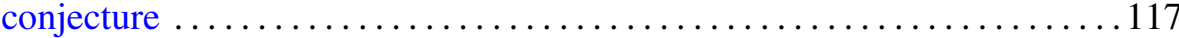

Shahriar Shahriari, On central type factor groups $\ldots \ldots \ldots \ldots \ldots \ldots \ldots 151$

Lynne Walling, Hecke eigenforms and representation numbers of quadratic forms ............................................... 179 